\title{
Functional Anatomy of Human Procedural Learning Determined with Regional Cerebral Blood Flow and PET
}

\author{
Scott T. Grafton, ${ }^{1,2,3}$ John C. Mazziotta, ${ }^{1,2,3}$ Sharon Presty, ${ }^{4}$ Karl J. Friston, ${ }^{5}$ Richard S. J. Frackowiak, ${ }^{5}$ and \\ Michael E. Phelps ${ }^{1,3}$ \\ 1Division of Nuclear Medicine and Biophysics, ${ }^{2}$ Department of Neurology, and ${ }^{3}$ Laboratory of Nuclear Medicine \\ (Department of Energy), U.C.L.A. School of Medicine, Los Angeles, California 90024, 'Department of Neurosurgery, \\ University of Virginia, Charlottesville, Virginia 22901, and ${ }^{5}$ MRC Cyclotron Unit, Hammersmith Hospital, London, United \\ Kingdom
}

\begin{abstract}
The functional anatomy of motor skill acquisition was investigated in six normal human subjects who learned to perform a pursuit rotor task with their dominant right hand during serial positron emission tomography (PET) imaging of relative cerebral blood flow (relCBF). The effect of motor execution, rather than learning, was identified by a comparison of four motor performance scans with two control scans (eye movements only). Motor execution was associated with activation of a distributed network involving cortical, striatonigral, and cerebellar sites. Second, the effect of early motor learning was examined. Performance improved from $17 \%$ to $66 \%$ mean time on target across the four PET scans obtained during pursuit rotor performance. Across the same scans, significant longitudinal increases of relCBF were located in the left primary motor cortex, the left supplementary motor area, and the left pulvinar thalamus. The results demonstrate that changes of regional cerebral activity associated with early learning of skilled movements occur in sites that are a subset of a more widely distributed network that is active during motor execution.
\end{abstract}

Humans demonstrate an impressive ability to acquire a vast repertoire of complex motor skills. Knowledge of the neural systems that are required for motor control and task execution has grown rapidly (Brooks, 1986). However, the mechanisms by which these systems facilitate the different stages of motor learning, and the precise location of synaptic change during learning are less well understood. In this study, we were interested in the functional anatomy of the initial stages of motor skill acquisition. It was hypothesized that this period of learning would be associated with dynamic alterations of cerebral activity in cortical motor areas that normally plan and execute motor programs. Putative sites would include the primary motor cortex, premotor areas, balsal ganglia, thalamus, and cerebellum. This hypothesis was tested by examining the temporal changes of relative cerebral blood flow (relCBF) acquired with serial positron emission tomography (PET) imaging in normal human

\footnotetext{
Received Sept. 26, 1991; revised Dec. 19, 1991 ; accepted Jan. 28, 1992.

We acknowledge Wendy Wilson for preparation of the illustrations. This work was supported in part by DOE Cooperative Agreement DE-FC03-87ER60615 and NIMH Grant RO1-MH-37916.

Correspondence should be addressed to Scott T. Grafton, M.D., Division of Radiological Sciences, University of Southern California, Clinical Sciences Center, Room 104, 2250 Alacazar Street, Los Angeles, CA 90033-4606.

Copyright (C) 1992 Society for Neuroscience $0270-6474 / 92 / 122542-07 \$ 05.00 / 0$
}

subjects as they learned to perform a pursuit rotor task. The study was designed to distinguish responses associated with execution of a visuomotor task from longitudinal changes associated with learning of the same skill.

\section{Materials and Methods}

\section{Subjects}

Six subjects participated in this study, in accordance with the Human Subjects Protection Committee of U.C.L.A. All of the subjects were U.C.L. $A$. undergraduates, recruited by advertisement, and identified as normal by an extensive medical and psychiatric interview and physical examination. Their mean age was $24( \pm 7)$, and the male : female proportion was $4: 2$. All were right handed as determined with a standardized questionnaire (Raczkowski and Kalat, 1980).

\section{Motor learning paradigm}

PET images of relative cerebral blood flow (relCBF) were obtained while subjects learned to perform a visually guided motor tracking task with a pursuit rotor device (Lafayette Instruments no. 30010). The device is a rotating $20 \mathrm{~cm}$ black disk with adjustable speed, similar to a small turntable. A $2 \mathrm{~cm}$ metal target is located on the periphery of the disk. The subject was instructed to keep the tip of a metal stylus against the moving target during trials of fixed duration. Subjects lay supine, with their head in the tomograph aperture. The pursuit rotor apparatus was positioned vertically in clear view and within comfortable reach so that the subjects could easily track the target throughout the entire excursion of the disk. Effects of learning were measured by the duration of time the stylus was on the target. Validation of this instrument for measuring visuomotor skill learning has been reported in detail over the past 40 years (Ammons, 1947; Ammons et al., 1958; Wisner et al., 1988). The two following behavioral conditions were used during PET imaging.

Pursuit condition. The subject held the stylus tip against the target on the disk of the pursuit rotor apparatus, beginning $10 \mathrm{sec}$ prior to isotope administration and imaging. Ten seconds after simultaneous isotope administration and dynamic imaging, the disk began to rotate at 60 rpm. The subject was instructed to try to keep the stylus on target. The pursuit task was performed for $80 \mathrm{sec}$ during imaging. The condition was performed during the second through fifth PET scans. No practice of the task had occurred prior to the second PET scan.

Control condition. Same as the pursuit condition, except that the subjects were instructed not to move their arm and to track the metal target of the disk with only their eyes. They kept the stylus stationary in the center of the disk. This task was performed during the first and sixth PET scans.

There was a 10-15 min interval between PET scans. After completion of the second, third, fourth, and fifth PET scan, the subject performed a structured practice session to accelerate improvement of pursuit performance (Fig. 1). Each practice session consisted of a 2 min rest period followed by seven trials lasting $20 \mathrm{sec}$, with a $20 \mathrm{sec}$ rest between successive trials. In addition, there was a $1 \mathrm{~min}$ intertrial rest after the third trial. Performance was monitored during the performance conditions and practice sessions by measuring the time the stylus was on target with an automatically triggered digital clock (Lafayette Instruments no. 54025). 


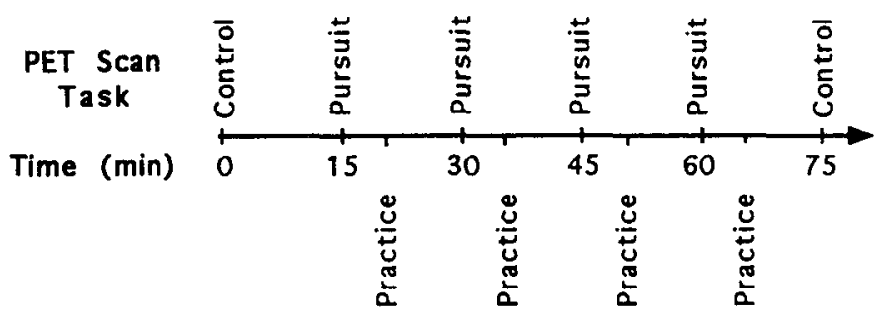

Figure 1. Experimental and imaging sequence of procedural learning.

\section{Imaging}

Each subject received six intravenous bolus injections of $50 \mathrm{mCi}$ of $\mathrm{H}_{2}{ }^{15} \mathrm{O}$ commensurate with dynamic positron emission tomographic imaging with the Siemens/CTI 831-08 tomograph (Siemens Corporation, Hoffman Estates, IL). The device collects 15 contiguous planes, 6.75 $\mathrm{mm}$ thick, with an in-plane resolution of $6 \mathrm{~mm}$ full width at halfmaximum (FWHM). Each sinogram of projection data was analyzed using a boundary detection algorithm, and emission scans were reconstructed using a calculated attenuation correction method (Bergstrom et al., 1982). Head immobilization was achieved with a custom foam mold (Smithers Corporation, Akron, $\mathrm{OH}$ ). The tomograph was oriented $10^{\circ}$ steeper than the canthomeatal line (i.e., frontal elevation/occipital depression) so that the field of view would extend from the vertex of the brain to the anterior cerebellum. Therefore, the orbital frontal lobes were not included.

Estimates of relCBF were based on a modified autoradiographic method (Herscovitch et al., 1983; Raichle et al., 1983). Arterial blood sampling was not performed. Five second frames were collected for $2 \mathrm{~min}$ beginning at the time of injection of $\mathrm{H}_{2}{ }^{15} \mathrm{O}$. The first frame showing the arrival of cerebral radioactivity was identified. From this time point the counts for the ensuing $70 \mathrm{sec}$ were summed to generate 15 plane images of relCBF (Fox et al., 1984; Mazziotta et al., 1985a).

\section{Image analysis}

The 15 plane relCBF image set from each PET scan $(6.75 \mathrm{~mm}$ interplane distance) was anatomically transformed to the standard coplanar stereotaxic atlas of Talairach and Tournoux (1988). This was performed by using a series of least-squares minimization algorithms that resize the brain relative to a normal population of PET images and then reorient and reslice the data sets relative to the anterior-posterior commissural line. Then, each plane was resampled in a nonlinear fashion to reduce variation in brain shape. The assumptions and operational details of these procedures are described in Friston et al. (1991a). In the standard stereotaxic space, each pixel is $2 \mathrm{~mm}^{2}$ and the interplane distance is $4 \mathrm{~mm}$. To remove high spatial frequency noise and to account for small scale differences in functional anatomy from subject to subject, each image was smoothed using a Gaussian filter (FWHM $=10$ pixels $=20 \mathrm{~mm}$ ).

Differences in global CBF between scans were then removed using an analysis of covariance (ANCOVA) on a pixel by pixel basis as previously described (Friston et al., 1990). The final relCBF images from the ANCOVA assume a mean global CBF of $50 \mathrm{ml} / 100 \mathrm{gm} / \mathrm{min}$. All values of relCBF reported in the Results, figures, and tables have been adjusted for differences of global CBF by ANCOVA and are in units of $\mathrm{ml} / 100 \mathrm{gm} / \mathrm{min}$. Because of smoothing, the adjusted pixel values corresponded to a mean relCBF of a weighted spherical region of radius $10 \mathrm{~mm}$ centered on the pixel.

The significance of responses at each pixel were also determined as follows. The ANCOVA also generates six (one for each sequential PET scan) adjusted group means and associated error variance (calculated across the six subjects) on a pixel by pixel basis. To identify differences between PET scans, a one-way analysis of variance (ANOVA) with planned comparison of means was performed using a $t$ statistic and linear contrasts of the group means and associated error variance at each pixel (Snedecor and Cochran, 1989). The resultant $t$ distributions were transformed to the normal distribution, generating Gaussian statistical parametric maps of significance. The significance maps were thresholded to make a corrcction for multiple nonindependent comparisons (pixels) as previously described (Friston et al., 1991b). For a significance of $p<0.05$, the threshold was set to give an expected false positive evcry 20 planes, and for $p<0.01$ the threshold was one false positive every 100 planes. Significant pixels were then displayed in transverse slices, with the grid from the Talairach atlas superimposed for anatomic localization.

\section{PET scan comparisons}

Three planned comparisons of means were made using a $t$ test for linear contrasts in a one-way ANOVA (Snedecor and Cochran, 1989):

(1) Movement effect: a comparison of the four motor performance scans (scans 2-5) to the control condition with no arm movement (scans 1 and 6 ).

(2) Learning effect: a comparison of the four motor performance scans (scans 2-5) to each other. The four coefficients of the linear contrast werc dcrived from the corrcsponding performance (the mean time on target of the six subjects) that were measured at the time of each PET scan. Weighting with this linear contrast would identify any cerebral areas where the relCBF was different between the four performance conditions, proportional to the increase in the performance measure. The inverse of this comparison was also tested, to identify any sites where relCBF decreased as a function of task assimilation.

(3) Time effect: A comparison of the two control conditions was performed to determine if there was a time effect independent of any learning effect.

\section{Results}

\section{Movement execution}

Motor execution was associated with the activation of a widely distributed set of cerebral areas, summarized in Table 1. In all, 11 sites demonstrated distinct increases of relCBF that were greater than the control condition $(p<0.01$, after correction for multiple comparisons), as illustrated in Figure 2. These sites could be broadly categorized into motor and visual association areas.

Of the motor system sites, the greatest response was located in the left motor cortex. This response was large in size and distributed over the middle two-thirds of the left motor strip. The peak site of relCBF response during the pursuit rotor task was located between two sites, active during shoulder and finger movements, identified in a previously reported investigation of the somatotopic organization of relCBF responses in the motor cortex (Colebatch et al., 1991).

The right motor cortex, ipsilateral to the performing limb, also demonstrated significant activation of relCBF, but of a lesser magnitude. This site was located within $8 \mathrm{~mm}$ of a site previously shown to be active during isolated shoulder movements (Colebatch et al., 1991).

Responses within the left supplementary motor area (SMA) were located in a bimodal distribution, with the peaks situated one above the other and separated by $8 \mathrm{~mm}$. The location of the two SMA sites were in proximity to sites identified during isolated movements of the index finger (superior site) and shoulder (inferior site) (Colebatch et al., 1991). In the right SMA, only the inferior site, the putative shoulder site, was significantly active.

Subcortical responses of the motor system were centered in the posterior portion of the left putamen and in the middle of the left substantia nigra. Because of effects of image smoothing, adjacent activation of the globus pallidus for the superior site or the red nucleus in the inferior site could not be excluded.

There was a large increase of relCBF throughout the middle and right parasaggital zones of the cerebellum extending from the anterior lobe to the inferior vermis. Within this extensive response site, local maxima were identified in the anterior lobe and the inferior vermis. Neither of the response maxima were centered over a cerebellar nucleus. Activation of a cerebellar 
Table 1. Changes of CBF during rotor pursuit

\begin{tabular}{llrlr} 
& $\begin{array}{l}\text { \% } \\
\text { Increase } \\
\text { in flow }\end{array}$ & \multicolumn{3}{l}{$\begin{array}{l}\text { Talairach } \\
\text { coordinates }^{a}\end{array}$} \\
\cline { 3 - 6 } Region & $9.5(3.3)$ & 24 & -22 & 56 \\
\hline Left motor cortex & $6.1(2.8)$ & -24 & -18 & 56 \\
Right motor cortex & $6.7(2.4)$ & 8 & -14 & 56 \\
Left superior SMA & $5.9(2.7)$ & 8 & -18 & 48 \\
Left inferior SMA & $4.8(1.8)$ & -4 & -16 & 48 \\
Right SMA & $2.2(1.3)$ & 28 & -20 & 0 \\
Left putamen & $2.3(0.7)$ & 10 & -18 & -8 \\
Left midbrain & $4.8(3.3)$ & -2 & -54 & -4 \\
Right anterior cerebellum & $8.9(2.6)$ & -4 & -52 & -20 \\
Right inferior vermis & $7.7(3.9)$ & -26 & -90 & 4 \\
Right extrastriate area 18 & $4.2(2.6)$ & 24 & -66 & 0 \\
Left fusiform gyrus & &
\end{tabular}

Data show the magnitude and location of significant relCBF responses associated with right hand rotor pursuit. The percentage increase and SD (in parentheses) of relCBF was calculated from a comparison of four pursuit rotor scans versus two control scans (eye movements only).

- Talairach coordinates are in millimeters, measured from the anterior end of the anterior-posterior commissural line, corresponding to the atlas of Talairach and Tournoux (1988).

nucleus may have been present but obscured due to the effects of image smoothing. We could not exclude additional local maxima in the inferior cerebellar hemisphere because it was not fully sampled.

There were two response sites of the visual system in the occipital lobes during execution of the pursuit rotor task. The first was located in the left fusiform gyrus and was less than 6 $\mathrm{mm}$ from a site previously identified as $\mathrm{V} 4$ in experiments of color vision (Lueck et al., 1989; Zeki et al., 1991). The second occipital response was located in the right extrastriate cortex, in the approximate region of Brodmann's area 18. This site was large and extended in a superior to inferior band, sparing the striate cortex. The site was distinct from and located inferior and posterior to a functional area previously identified as V5 in human studies of visual motion detection (Zeki et al., 1991).

\section{Motor learning}

Each of the subjects successfully learned to perform the pursuit rotor task over the course of the experiment. Performance changed from jerky movements with ballistic corrections in the first trial to smooth continuous tracking by the last trial. The duration of time that the stylus was on the target of the pursuit rotor apparatus was used to determine motor performance as demonstrated in Figure 3. The time on target was significantly different between performance trials 1 and 2 , and between trials 2 and 3, confirming the presence of a learning effect (paired $t$ test, $p<0.05$ ). The greatest change in performance was between the first and second performance trials, showing that the learning effect developed rapidly. No gender effects were present.

The four PET scans of motor performance were compared with each other to determine sites of increasing relCBF. Three sites were identified: the left SMA, the left motor cortex, and the left thalamus $(p<0.05$, after correction for multiple comparisons by one-way ANOVA and linear contrasts), as demonstrated in Figure 4. The location, magnitude, and percentage change of relCBF of these sites are summarized in Table 2. The motor cortex site was located within $2 \mathrm{~mm}$ of the same peak

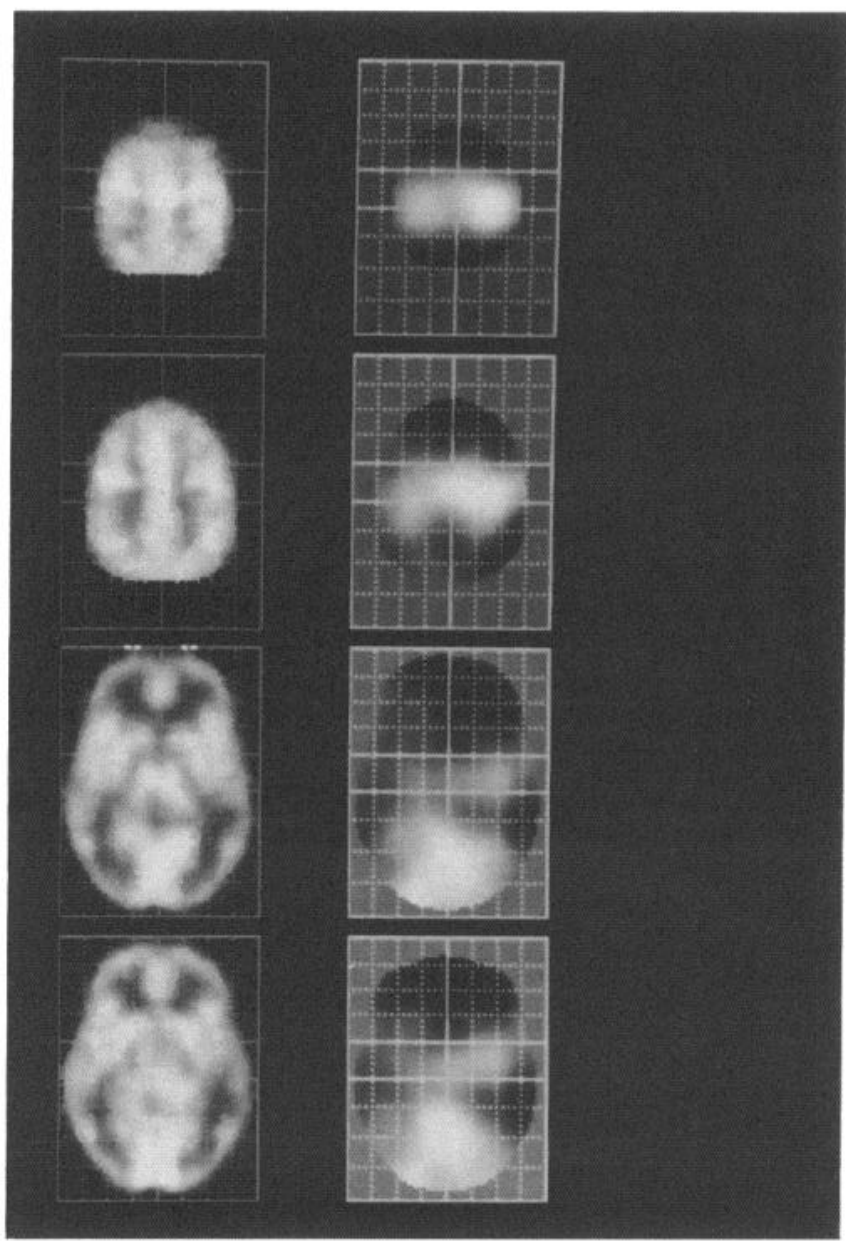

Figure 2. The effect of movement in the pursuit rotor task. The first column demonstrates relCBF after stereotaxic normalization at four brain levels and serves as an anatomic reference. The second column demonstrates the absolute difference of relCBF of all six subjects between the four performance and two control conditions at the same brain levels. Only increased relCBF is shown in white in the difference images. Significant increases of relCBF were centered in the bilateral primary motor cortices, two sites of the SMA, the posterior left putamen, the left midbrain, multiple sites of the cerebellum, the right fusiform gyrus, and left Brodmann's area 18 of the occipital lobes. The four rows correspond to $56,48,0$, and $-8 \mathrm{~mm}$ relative to the anterior-posterior commissural line as defined in the atlas of Talairach and Tournoux (1988). The subjects' left hemisphere is on the right side of the image.

present during motor execution. The SMA peak associated with a learning effect was located midway between the two SMA peaks associated with motor execution. The thalamic site was centered in the left pulvinar. When a comparison was made between only the first and last pursuit trials by $t$ test, the same three areas continued to demonstrate a significant difference.

Responses from each individual at these sites are shown in Figure 5. All three areas demonstrate a rapid rise in relCBF after the first pursuit trial. Only the SMA showed persistent increases of relCBF across all trials, similar to the performance curve shown in Figure 3. No gender effects are noted in the relCBF responses.

An analysis was also performed to determine if there was any cerebral site with a significant decrease of relCBF during motor learning. No significant sites were detected. 


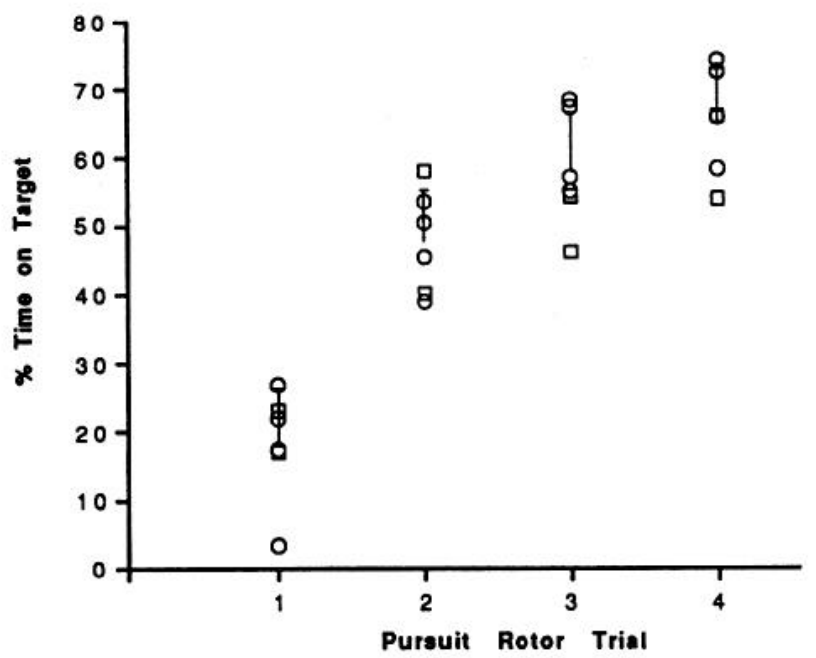

Figure 3. A measure of performance (percentage of time on target) for each pursuit rotor trial demonstrates a learning effect across trials. Differences were significant between trials 1 and 2 , and 2 and $3(p<0.05$, paired $t$ test). No gender differences were present. Circles, male; squares, female; error bars, one-sided SD.

\section{Time effect}

A comparison of the two control conditions, obtained at the start and completion of PET scanning, demonstrated no significant differences of relCBF in any brain region over the course of the experiment. Thus, there was no evidence of a time effect to suggest habituation developing independently of motor execution.

\section{Discussion}

Motor skill acquisition is a fundamentally important operation of the nervous system. In this study, the functional anatomy of motor learning was clearly distinguished from the anatomy of simple motor execution. The principal findings were longitudinal increases in neuronal activity in the left motor cortex, left SMA, and pulvinar thalamus during motor skill assimilation. In contrast, a more widely distributed network involving cortex, basal ganglia, and cerebellum was active during motor execution. These results support the hypothesis that early procedural learning occurs within a subset of the neuronal network where the behavior is actualized.

In learning a motor skill, a performer must combine the appropriate movements into the correct pattern in both spatial and temporal domains. The awkward, jerky movements seen in the earliest stages of motor learning can be accounted for by

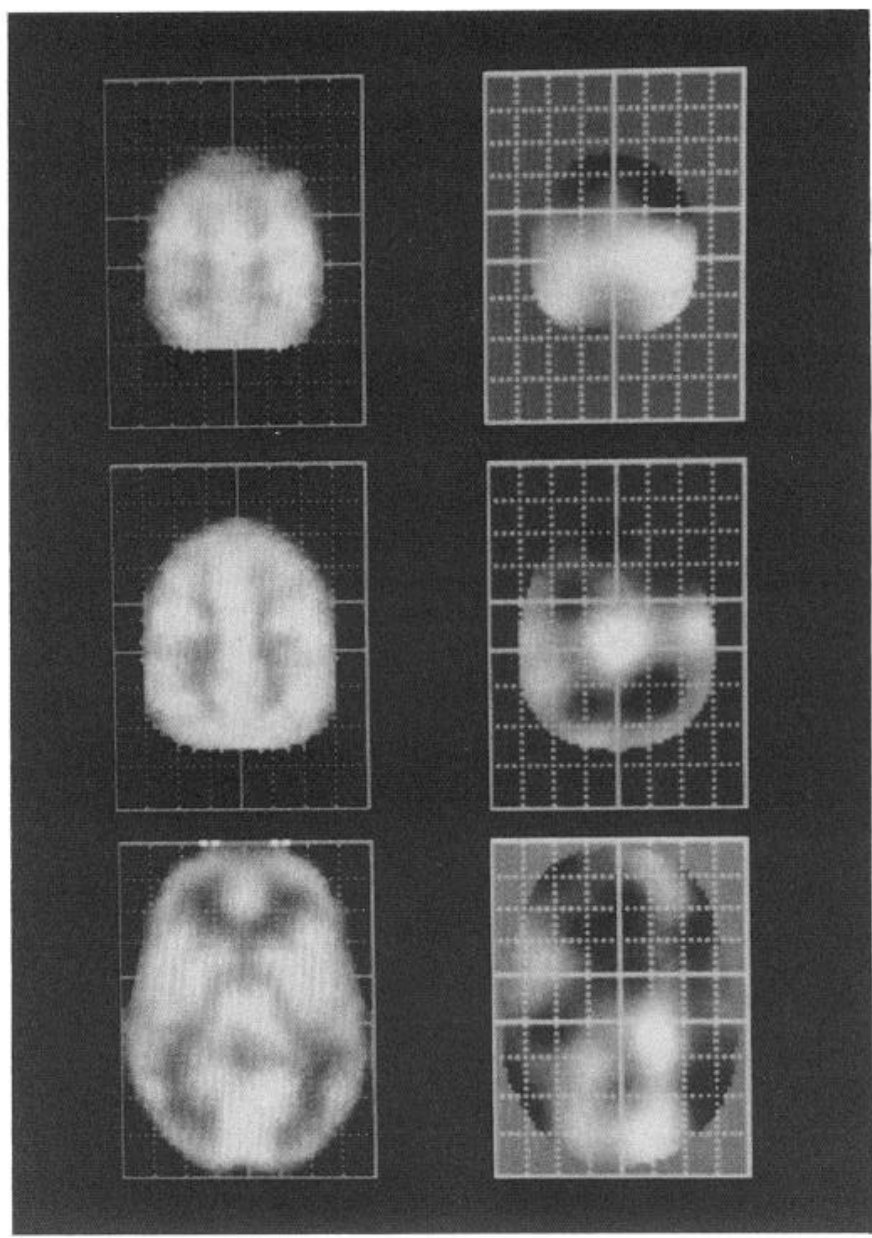

Figure 4. The effect of learning the pursuit rotor task. The first column demonstrates relCBF at three brain levels after stereotaxic normalization and serves as an anatomic reference. The second column demonstrates the absolute difference of relCBF for all six subjects across the four performance conditions at the same brain levels. Only pixels with increasing relCBF across performance conditions are shown in white in the difference images. Significant increases of relCBF were centered in the left SMA, primary motor cortex, and pulvinar thalamus. The levels correspond to 56,48 , and $0 \mathrm{~mm}$ above the anterior-posterior commissural line as defined in the atlas of Talairach and Tournoux (1988). The subjects' left hemisphere is on the right side of the image.

feedback systems that rely on visually perceived errors to generate corrective motor commands. After practice, movements become smooth, coordinated, and often more rapid, suggesting acquisition and modification of motor programs that allow the

Table 2. CBF changes during motor skill learning

\begin{tabular}{|c|c|c|c|c|c|c|c|c|}
\hline \multirow[b]{2}{*}{ Region } & \multicolumn{3}{|c|}{ Talairach coordinates ${ }^{a}$} & \multicolumn{4}{|c|}{ relCBF during rotor pursuit } & \multirow{2}{*}{$\begin{array}{l}\% \text { Increase of } \\
\text { flow between } \\
\text { trials } 1 \text { and } 4\end{array}$} \\
\hline & $\mathrm{x}$ & $\mathrm{y}$ & $\mathrm{z}$ & Trial 1 & Trial 2 & Trial 3 & Trial 4 & \\
\hline Left motor cortex & 24 & -24 & 56 & $61.1(1.4)$ & $64.8(1.4)$ & $64.6(2.0)$ & $64.8(1.1)$ & $6.1 \%(2.8)$ \\
\hline Left SMA & 2 & -14 & 52 & $64.2(0.7)$ & $65.4(0.8)$ & $66.0(1.4)$ & $67.0(0.4)$ & $4.5 \%(0.9)$ \\
\hline Left thalamus & 20 & -32 & 4 & $53.7(1.3)$ & $55.7(0.8)$ & $55.5(0.7)$ & $55.9(0.5)$ & $4.1 \%(2.6)$ \\
\hline
\end{tabular}

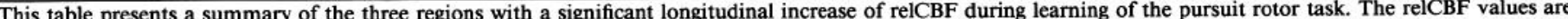

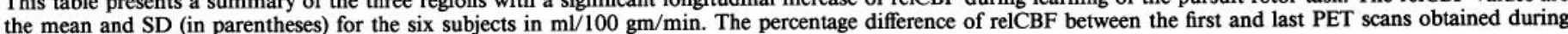
pursuit rotor tracking is also shown.

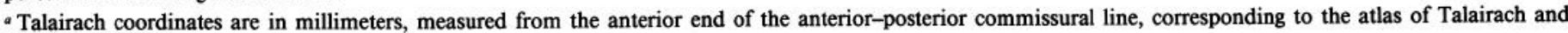
Tournoux (1988). 
a

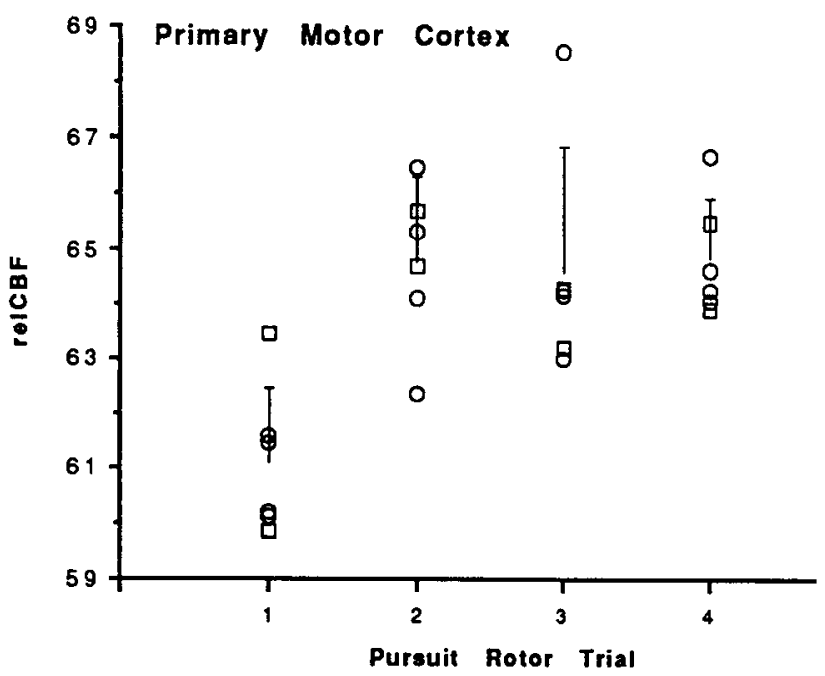

b

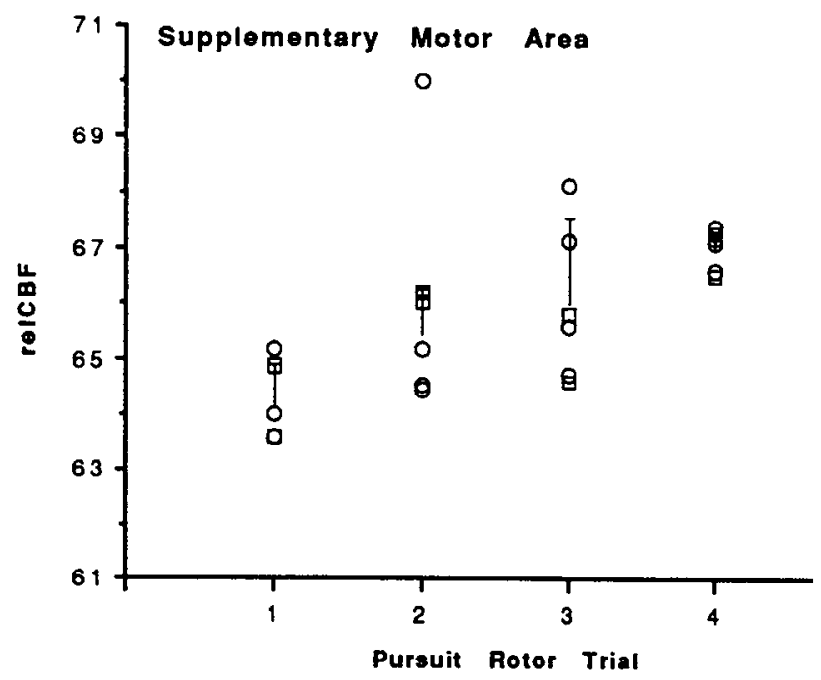

C

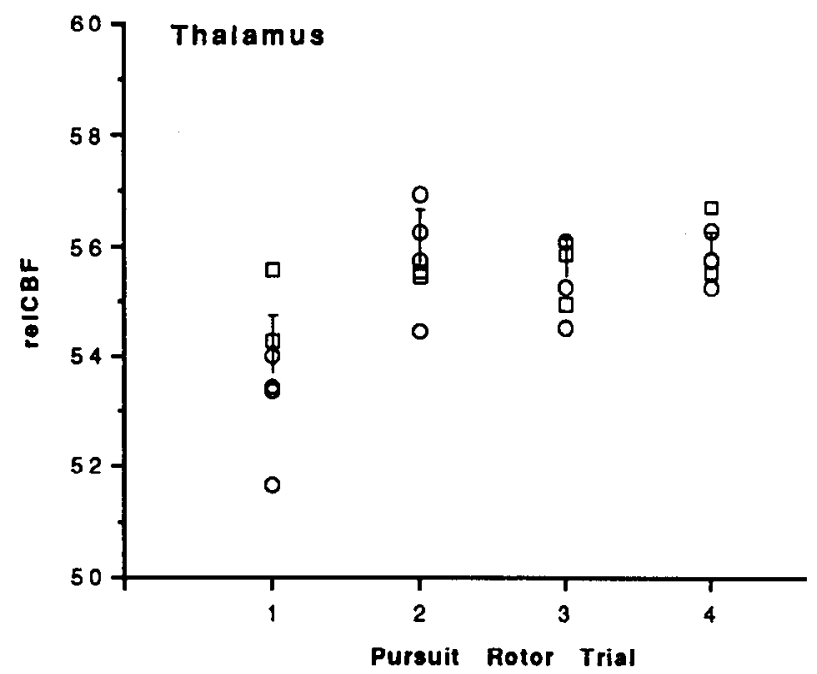

performer to organize a sequence of movements in advance of the execution, and with less reliance on visual feedback. With additional practice the skill becomes overlearned, automatic, or proceduralized. It has been proposed that, at the beginning of practice for a new motor skill, the performer adopts a strategy of constructing and facilitating subskills previously retained from other well-learned tasks (Eysenck and Frith, 1977). This process has becn termed the acquisition of set and likely occurs in the first stages of learning where there are the most rapid improvements of performance. In our results, these rapid changes occurred most dramatically between the first and second trials, when there was a concomittant increase of neuronal activity in the SMA, motor cortex, and pulvinar thalamus.

Neuronal discharges of the SMA typically precede motor cortex activity and also are active during motor ideation, independent of performance (Orgogozo and Larsen, 1979; Roland et al., 1980; Deecke et al., 1985). This would suggest an executor function over the primary motor cortex for the SMA, in which the conception and initiation of movement take place. The findings herein are consistent with this functional description of the SMA and also imply that the SMA may be critical for execution of previously learned motor subskills or acquisition of motor set.

All of the subjects used less corrective movements, and acquired smoother movements with each successive performance trial. It is unlikely, therefore, that the increased relCBF in the primary motor cortex was secondary to an increased rate of movement or an increased rate of change of limb direction. We would propose that the increase of relCBF in the motor cortex is due, in part, to the selection of a discrete set of motor neurons that are an approximation of the optimal set required for task execution. The specific changes in neuronal processing in the motor cortex during this stage cannot be determined with our study. CBF is thought to refiect the discharge rate at the presynaptic terminal. It is possible that the increase in motor cortex during skill acquisition reflects, in part, increased firing of afferents from the SMA or thalamus. We explicitly examined both augmentation and attenuation of relCBF associated with improvement in performance. Electrophysiological studies of motor learning in animals have implicated both long-term potentiation and long-term depression (in the cerebellum) as modulators of synaptic activity (Sakamoto et al., 1987; Asanuma, 1989; Ito, 1989). Longitudinal changes of relCBF may be the neurophysiological correlates of adaptation in neural discharge rates that underlie skill acquisition.

The increased responses of relCBF identified in the pulvinar thalamus are more difficult to interpret, given the diversity of complex systems that are presumed to be integrated in this nucleus. Recent studies have demonstrated that neurons in the inferior and lateral pulvinar of nonhuman primates are visual in nature and modulated by a number of eye movement conditions (Bender, 1981). Robinson has proposed that signals from the pulvinar might be used for attentional purposes and the triggering of motor responses (Robinson et al., 1991). In humans, pulvinar activation has been demonstrated with changes

Figure 5. The responses of relCBF (in $\mathrm{ml} / 100 \mathrm{gm} / \mathrm{min}$ ) in the left primary motor cortex $(a)$, left SMA $(b)$, and left thalamus $(c)$ of each individual during pursuit rotor learning. Differences were significant across the four scans at each site by ANOVA. No gender effects were present. Circles, male; squares, female; error bars, one-sided SD. 
in visual attention in a PET study using measures of regional glucose metabolism (LaBerge and Buchsbaum, 1990). For the pursuit rotor task, the visual system must generate a stable image of the pursuit target in anticipation of any motor system output. We would propose that the change in pulvinar activity across trials was secondary to a shift in the visual attention given to the pursuit target. Since we did not measure eye movements, we cannot correlate the pulvinar activity to spccific eye movement parameters across trials.

Other components of a large corticostriatonigral-cerebellar network were active during the pursuit rotor task, although they did not change during the learning process. These sites have all been shown previously to be critical for execution of other types of motor behavior with a variety of anatomic and physiologic methods. The functional anatomy of this network forms the basis for a number of models of motor control (Brooks, 1986). In addition, the somatotopic organization of the motor cortex and SMA observed in this study reiterates several previous PET experiments (Colebatch et al., 1991; Grafton et al., 1991). The somatotopic organization of the frontal lobe is exceptionally complex, with multiple maps and overlapping cortical fields (Wise and Strick, 1984; Strick, 1988). Despite this complexity, noted with single neuron recordings, the PET results have consistently demonstrated a large-scalc homuncular distribution in both the motor cortex and SMA. In the present study, it was notable that the SMA response was bimodal, with maxima located in the putative hand and shoulder areas. This is consistent with the types of movements at multiple joint segments that were required of this task.

It should be reiterated that the lack of a change in the magnitude of relCBF over time in these other corticostriatonigralcerebellar sites does not imply that they are not involved in the learning of a motor task. Instead, each site is a critical component of an integrated system specifically designed to incorporate new motor plans. Clinical observations from patients and nonhuman primates with injury to the striatum or cerebellum confirm that the entire loop must be intact for the motor skill to be assimilated (Mishkin et al., 1984; Inhoff et al., 1989; Halsband and Freund, 1990; Sanes et al., 1990). For example, the striatal dopamine deficiency of Parkinson's disease lcads to impaired procedural learning (Harrington et al., 1990). Of note, these patients fail to activate the SMA during motor task execution (Playford et al., 1991). Premotor and primary motor cortex responses remain normal, however. Integrity of the putamen-SMA projections is likely critical for both performance and motor plan assimilation.

The cerebellum has long been described as a "teaching machine" of the motor cortex (Marr, 1969; Ito, 1972; Thach, 1978). Thus, it was interesting to find no longitudinal change of activity in this structure during motor skill learning in our study. Attenuation of relCBF responses in the cerebellum and SMA has been observed during the learning of a simple finger opposition algorithm (Friston et al., 1991c). These paradigms are markedly different in design, and their differences may provide insight into different aspects of motor learning. We explicitly studied the carly phascs of skill acquisition, placing a strong emphasis on acquisition of motor set dependent on visual feedback (the onset of performance coincided with scanning). By the end of the session, complete automaticity had not been achieved. In contrast, the finger tapping paradigm emphasized automaticity of a sequential pattern, paced with an auditory cue. These findings are consistent with the notion that supratentorial systems are involved in the acquisition and optimization of set in early learning whereas other systems, including cerebellum, may subtend consolidation with practice. That is, consolidation may involve the transfer of function between and within neural networks normally active during motor execution (Mazziotta et al., 1985b). To test this further, experiments are in progress to examine the functional anatomy of the pursuit rotor task after long-term practice.

To analyze the PET data, we used ANOVA with weighted comparisons of means by linear contrasts. This is a novel application of a basic statistical method for determining functional change that goes beyond a simple categorical or subtractive approach for interpreting changes in brain physiology. This method is a logical extension of the basic $t$ test and allows for a comparison of more than two groups at a time. In addition, the technique is a valid method for weighting relCBF values by corresponding performance data. We have used weights (the linear contrasts) that are continuous variables reflecting a key aspect of motor performance. The statistical approach should be useful in analyzing a variety of longitudinal PET experiments in which a continuous variable of performance or rate can be simultaneously acquired.

\section{References}

Ammons RB (1947) Acquisition of motor skills. II. Rotary pursuit performance with continuous practice before and after a single rest. J Exp Psychol 37:393-411.

Ammons RB, Farr RG, Bloch E, Neumann E, Dey M, Marion R, Ammons CH (1958) Long-term retention of perceptual-motor skills. J Exp Psychol 55:3318-3328.

Asanuma H (1989) The motor cortex. New York: Raven.

Bender DB (1981) Retinotopic organization of the macaque pulvinar. J Neurophysiol 46:672-693.

Bergstrom M, Litton J, Eriksson L, Bohm C, Blomqvist G (1982) Determination of object contour from projections for attenuation correction in cranial positron emission tomography. J Comput Assist Tomogr 6:365-372.

Brooks VB (1986) The neural basis of motor control. New York: Oxford UP

Colebatch JG, Deiber M-P, Passingham RE, Friston KJ, Frackowiak RSJ (1991) Regional cerebral blood flow during voluntary arm and hand movements in human subjects. J Neurophysiol 65:1392-1401.

Deecke L, Komhuber HH, Lang W, Lang M, Schreiber H (1985) Timing function of the frontal cortex in sequential motor and learning tasks. Human Neurobiol 4:143-154.

Eysenck HJ, Frith CD (1977) Reminiscence, motivation, and personality. New York: Plenum.

Fox PT, Mintun MA, Raichle ME, Herscovitch P (1984) A noninvasive approach to quantitative functional brain mapping with $\mathrm{H}_{2}{ }^{15} \mathrm{O}$ and positron emission tomography. $\mathrm{J}$ Cereb Blood Flow Metab 4 329-333.

Friston KJ, Frith CD, Liddle PF, Dolan RJ, Lammertsma AA, Frackowiak RSJ (1990) The relationship between global and local changes in PFT scans. J Cereb Blood Flow Metab 10:458-466

Friston KJ, Frith CD, Liddle PF, Frackowiak RSJ (1991a) Plastic transformation of PET images. J Comput Assisted Tomogr 15:634639.

Friston KJ, Frith CD, Liddle PF, Frackowiak RSJ (1991b) Comparing functional (PET) images: the assessment of significant change. J Cereb Blood Flow Metab 11:690-699.

Friston KJ, Frith CD, Liddle PF, Frackowiak RSJ (1991c) The cerebellum in skill learning. J Cereb Blood Flow Metab [Suppl] 1 1:S440.

Grafton ST, Woods RP, Mazziotta JC, Phelps ME (1991) Somatotopic mapping of the primary motor cortex in man: activation studies with cerebral blood flow and PET. J Neurophysiol 66:735-743.

Halsband U, Freund H-J (1990) Promotor cortex and conditional motor learning in man. Brain 113:207-222.

Harrington DL, Haaland KY, Yeo RA, Marder E (1990) Procedural memory in Parkinson's disease: impaired motor but not visuoperceptual learning. J Clin Exp Neuropsychol 12:323-339. 
Herscovitch P, Markham J, Raichle ME (1983) Brain blood flow measured with intravenous $\mathrm{H}_{2}{ }^{15} \mathrm{O}$. I. Theory and error analysis. $\mathrm{J}$ Nucl Med 24:782-789.

Inhoff AW, Diener HC, Rafal RD, Ivry R (1989) The role of cerebellar structures in the execution of serial movements. Brain 112:565-581.

Ito M (1972) Neural design of the cerebellar motor control system. Brain Res 40:81-102.

Ito M (1989) Long-term depression. Annu Rev Neurosci 12:85-102.

LaBerge D, Buchsbaum MS (1990) Positron emission tomographic measurements of pulvinar activity during an attention task. J Neurosci 10:613-619.

Lueck CJ, Zeki S, Friston KJ, Deiber M-P, Cope P, Cunningham VJ, Lammertsma AA, Kennard C, Frackowiak RSJ (1989) The colour centre in the cerebral cortex of man. Nature 340:386-389.

Marr D (1969) A theory of cerebellar cortex. J Physiol (Lond) 202: $437-470$.

Mazziotta JC, Huang S-C, Phelps ME, Carson RE, MacDonald NS, Mahoney K (1985a) A noninvasive positron computed tomography technique using oxygen-15-labeled water for the evaluation of neurobehavioral task batteries. J Cereb Blood Flow Metab 5:70-78.

Mazziotta JC, Phelps ME, Wapenski JA (1985b) Human cerebral motor system metabolic responses in health and disease. J Cereb Blood Flow Metab [Suppl] 5:S213-S214.

Mishkin M, Malamut B, Bachevalier J (1984) Memories and habits: two neural systems. In: Neurobiology of learning and memory (Lynch G, McGaugh JL, Weinberger NM, eds), pp 65-77. New York: Guilford.

Orgogozo JM, Larsen B (1979) Activation of the supplementary motor area during voluntary movement in man suggests it works as a supramotor area. Science 206:847-850.

Playford ED, Passingham RE, Nutt J, Frackowiak RSJ, Brooks DJ (1991) Impaircd activation of medial frontal arcas during movement in Parkinson's disease: a PET study. J Cereb Blood Flow Metab 11: S1.

Rackowski D, Kalat JW (1980) Reliability and validity of some handedness questionnaire items. Neuropsychologia 18:213-217.
Raichle ME, Martin WRW, Herscovitch P (1983) Brain blood flow measured with intravenous $\mathrm{H}_{2}{ }^{15} \mathrm{O}$. II. Implementation and validation. J Nucl Med 24:790-798.

Robinson DL, McClurkin JW, Kertzman C, Petersen SE (1991) Visual responses of pulvinar and collicular neurons during eye movements of awake, trained macaques. J Neurophysiol 11:485-496.

Roland PE, Larsen B, Lassen NA, Skinhøj E (1980) Supplementary motor area and other cortical areas in organization of voluntary movements in man. J Neurophysiology 43:118-136.

Sakamoto T, Porter LL, Asanuma $\mathrm{H}$ (1987) Long-lasting potentiation of synaptic potentials in the motor cortex produced by stimulation of the sensory cortex in the cat: a basis of motor learning. Brain Res 413:360-364.

Sanes JN, Dimitrov B, Hallett M (1990) Motor learning in patients with cerebellar dysfunction. Brain 113:103-120.

Snedecor GW, Cochran WG (1989) Statistical methods. Ames: Iowa State UP.

Strick PL (1988) Anatomical organization of multiple motor areas in the frontal lobe: implications for recovery of function. In: Advances in neurology (Waxman SG, ed), pp 293-312. New York: Raven.

Talairach J, Tournoux P (1988) Co-planar stereotaxic atlas of the brain. New York: Thieme.

Thach WT (1978) Correlation of neural discharge with pattern and force of muscular activity, joint position, and direction of intended next movement in motor cortex and cerebellum. J Neurophysiol 41: 654-676.

Wise SP, Strick PL (1984) Anatomical and physiological organization of the non-primary motor cortex. Trends Neurosci 8:442-446.

Wisner BL, Lombardo JP, Catalano JF (1988) Rotary pursuit performance as a function of sex, sex-role, and intertrial interval. Percept Motor Skills 66:443-452.

Zcki S, Watson JDG, Lucck CJ, Friston KJ, Kennard C, Frackowiak RSJ (1991) A direct demonstration of functional specialization in human visual cortex. J Neurosci 11:641-649. 